\title{
Computational Study of the Different Waverider Configurations Aerodynamics
}

\author{
D.S. Yatsukhno \\ Ishlinsky Institute for Problems in Mechanics of the Russian Academy of Sciences, \\ Moscow, 119526, Russia \\ yatsukhno-ds@rambler.ru
}

\begin{abstract}
The current paper contains the elliptic cone waverider and inclined cone waverider aerodynamics calculations results. The comparison between the numerical and experimental data was performed for the wide range of the angles of attack and sleep angles. All results was obtained by the UST3D code using different Riemann solvers within the framework of the splitting method. The acceptable agreement between the present results, the thin-layer Navier-Stokes and also parabolized Navier-Stokes data was demonstrated.
\end{abstract}

Keywords: finite volume method, splitting methods, Riemann solvers, waveriders

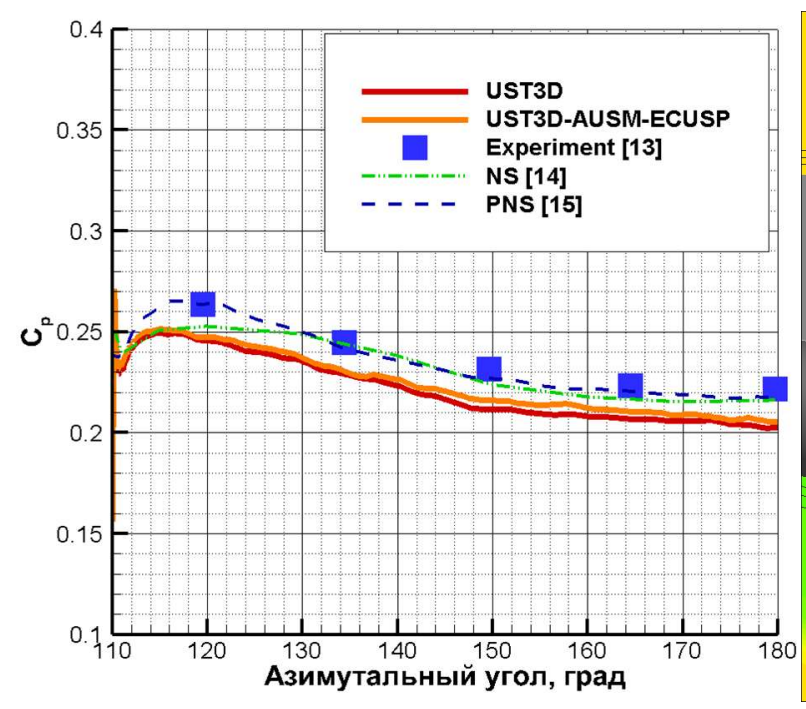

Comparison between the numerical and experimental pressure coefficient data for elliptic cone waverider at $\mathrm{M}_{\infty}=4$ under zero angle of attack

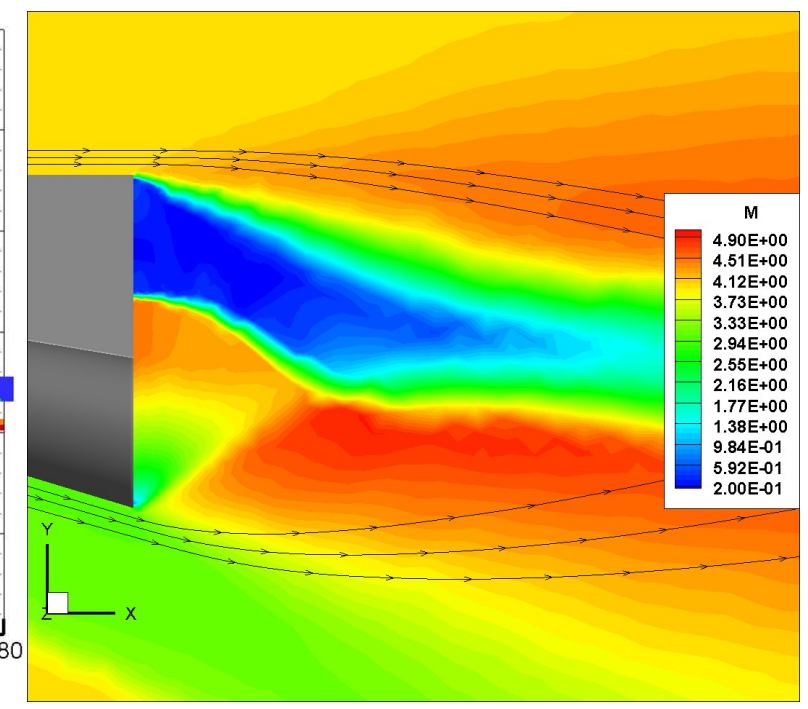

Elliptic cone waverider base flow and streamlines obtained using AUSM-ECUSP scheme at $\mathrm{M}_{\infty}=4$ and $\alpha=0^{\circ}$

\section{Freestream conditions}

\begin{tabular}{|c|c|}
\hline Parameter & Value \\
\hline Pressure $p_{\infty}, \mathrm{erg} / \mathrm{cm}^{3}$ & 14530 \\
\hline Density $\rho_{\infty}, \mathrm{g} / \mathrm{cm}^{3}$ & $0.546 \times 10^{-4}$ \\
\hline Temperature $T_{\infty}, \mathrm{K}$ & 92.8 \\
\hline Mach number $\mathrm{M}_{\infty}$ & 4 \\
\hline
\end{tabular}




\title{
Численное моделирование аэродинамики волнолетов построенных на скачках уплотнения различной формы
}

\author{
Д.С. Яцухно \\ Институт проблем механики им. А.Ю. Иилинского Российской академии наук, \\ Россия, Москва, 119526, проспект Вернадского, д. 101, корп. 1 \\ yatsukhno-ds@rambler.ru
}

\begin{abstract}
Аннотация
В рамках настоящей работы представлены результаты систематических расчетов аэродинамических характеристик высокоскоростных летательных аппаратов - волнолетов, построенных на ударных волнах, образующихся при обтекании эллиптического или наклонного кругового конуса. Выполнено сравнение результатов численного моделирования с экспериментальными данными, полученными для различных углов атаки и скольжения. Продемонстрированы особенности совместной реализации метода расщепления по физическим процессам и одного из вариантов AUSM схемы. Проведено сравнение результатов расчетного исследования, осуществленного с использованием компьютерного кода UST3D и его модификации, со сторонними расчетными данными по аэродинамике различных конфигураций волнолетов, полученных с применением структурированных сеток, уравнений Навье-Стокса в приближении тонкого слоя, а также параболизованных уравнений Навье - Стокса.
\end{abstract}

Ключевые слова: метод контрольного объема, расщепление по физическим процессам, распад произвольного разрыва, аэродинамика волнолетов

\section{1. Введение}

Интерес к исследованию волнолетов обусловлен потенциально высокими аэродинамическими характеристиками, присущими данной конфигурации. Облик волнолета в первую очередь зависит от контура его передней кромки и геометрии ударной волны, возмущенное течение за которой является основой несущей части высокоскоростного летательного аппарата (ВЛА). При этом форма скачка уплотнения позволяет получать как плоские, так и криволинейные поверхности волнолета. Перечисленные особенности позволяют сделать вывод о многообразии форм волнолетов, что находит отражение в существенном количестве публикаций, посвященных расчетным и экспериментальным исследованиям аэродинамики и теплообмена данного вида ВЛА [1-7].

Как отмечалось ранее, для определения формы волнолета, фактически, необходимо осуществлять расчет течения за ударной волной. Для случая прямого кругового конуса этот процесс может быть сведен к численному интегрированию системы обыкновенных дифференциальных уравнений [8]. Если анализируется течение за эллиптическим скачком уплотнения с малым эксцентриситетом тела-генератора ударной волны или рассматривается обтекание конуса сверхзвуковым потоком под малым углом атаки, то в качестве основного подхода могут быть использованы методы гиперзвуковой теории малых возмущений, которые позволяют получить приближенное решение интегрального уравнения, сформулированного относительно функции тока [9]. В работах были [9-11] выполнены расчетно-теоретические исследования подобных течений, а в работах [12-15] полученные аналитические 
выражения применялись для описания несущих поверхностей волнолетов, для которых впоследствии был проведен ряд стендовых экспериментов [13], а также численное моделирование с применением различных компьютерных кодов [14-15]. Сравнение с упомянутыми данными и является основной целью настоящей работы.

\section{2. Постановка задачи}

Результаты систематического применения метода расщепления по физическим процессам для решения задач вычислительной аэродинамики изложены в работах [16-20]. Вычислительный инструментарий, реализованный в компьютерном коде UST3D [16], позволяет выполнять численное моделирование процессов обтекания ВЛА сложной формы с использованием неструктурированных тетраэдральных сеток без необходимости выделения ударных волн. Приближенное решение задачи о распаде произвольного разрыва осуществляется с применением схемы с донорными ячейками [21], адаптированной к использованию в рамках метода контрольного объема.

В исходную систему интегрируемых уравнений входят: уравнение неразрывности, уравнения Навье - Стокса и уравнение энергии, представленных в векторной форме

$$
\frac{\partial \mathbf{w}}{\partial t}+\frac{\partial}{\partial x}\left(\mathbf{F}_{x}\right)+\frac{\partial}{\partial y}\left(\mathbf{F}_{y}\right)+\frac{\partial}{\partial z}\left(\mathbf{F}_{z}\right)=\frac{\partial}{\partial x}\left(\mathbf{G}_{x}\right)+\frac{\partial}{\partial y}\left(\mathbf{G}_{y}\right)+\frac{\partial}{\partial z}\left(\mathbf{G}_{z}\right),
$$

Компоненты вектора невязкого потока $\mathbf{F}_{x}, \mathbf{F}_{y}$ и $\mathbf{F}_{z}$ могут быть представлены в виде суммы конвективного потока и части, обусловленной изменением давления, вид которой зависит от консервативной переменной, входящей в уравнение энергии [22]

$$
\mathbf{F}_{x}=\left[\begin{array}{l}
\rho u \\
\rho u^{2} \\
\rho u v \\
\rho u w \\
\rho u E
\end{array}\right]+\left[\begin{array}{l}
0 \\
p \\
0 \\
0 \\
p u
\end{array}\right], \mathbf{F}_{y}=\left[\begin{array}{l}
\rho v \\
\rho u v \\
\rho v^{2} \\
\rho v w \\
\rho v E
\end{array}\right]+\left[\begin{array}{l}
0 \\
0 \\
p \\
0 \\
p v
\end{array}\right], \mathbf{F}_{z}=\left[\begin{array}{l}
\rho w \\
\rho w u \\
\rho w v \\
\rho w^{2} \\
\rho w E
\end{array}\right]+\left[\begin{array}{l}
0 \\
0 \\
0 \\
p \\
p w
\end{array}\right]
$$

Соотношение (2) также отражает смысл расщепления по физическим процессам - pacчет потоков, обусловленных конвекцией или давлением производится в два этапа. Компоненты вектора вязкого потока $\mathbf{G}_{x}, \mathbf{G}_{y}$ и $\mathbf{G}_{z}$ имеют следующий вид:

$$
\mathbf{G}_{x}=\left[\begin{array}{c}
0 \\
\tau_{x x} \\
\tau_{x y} \\
\tau_{x z} \\
u \tau_{x x}+v \tau_{x y}+w \tau_{x z}-q_{x}
\end{array}\right], \mathbf{G}_{y}=\left[\begin{array}{c}
0 \\
\tau_{y x} \\
\tau_{y y} \\
\tau_{y z} \\
u \tau_{y x}+v \tau_{y y}+w \tau_{y z}-q_{y}
\end{array}\right], \mathbf{G}_{z}=\left[\begin{array}{c}
0 \\
\tau_{z x} \\
\tau_{z y} \\
\tau_{z z} \\
u \tau_{z x}+v \tau_{z y}+w \tau_{z z}-q_{z}
\end{array}\right]
$$

Вектор консервативных переменных $\mathbf{w}$ имеет следующий вид:

$$
\mathbf{w}=\left[\begin{array}{lllll}
\rho & \rho u & \rho v & \rho w & \rho E
\end{array}\right]^{\mathrm{T}}
$$

В уравнениях (1)-(4) $\rho$ - плотность; $u, v, w$ - проекции вектора скорости; $E$ - удельная полная энергия; $p$ - давление; $q_{x}, q_{y}, q_{z}$ - составляющие вектора теплового потока. Компоненты тензора вязких напряжений определяются с помощью следующих соотношений:

$$
\tau_{x x}=\mu\left(\frac{4}{3} \frac{\partial u}{\partial x}-\frac{2}{3} \frac{\partial v}{\partial y}-\frac{2}{3} \frac{\partial w}{\partial z}\right), \tau_{y y}=\mu\left(\frac{4}{3} \frac{\partial v}{\partial y}-\frac{2}{3} \frac{\partial u}{\partial x}-\frac{2}{3} \frac{\partial w}{\partial z}\right),
$$




$$
\begin{gathered}
\tau_{z z}=\mu\left(\frac{4}{3} \frac{\partial w}{\partial z}-\frac{2}{3} \frac{\partial u}{\partial x}-\frac{2}{3} \frac{\partial v}{\partial y}\right), \tau_{x y}=\tau_{y x}=\mu\left(\frac{\partial v}{\partial x}+\frac{\partial u}{\partial y}\right), \\
\tau_{y z}=\tau_{z y}=\mu\left(\frac{\partial w}{\partial y}+\frac{\partial v}{\partial z}\right), \tau_{x z}=\tau_{z x}=\mu\left(\frac{\partial u}{\partial z}+\frac{\partial w}{\partial x}\right)
\end{gathered}
$$

Здесь $\mu$ - коэффициент динамической вязкости. Уравнение состояния совершенного газа и закон Фурье являются замыкающими соотношениями для исходной системы уравнений газовой динамики. Также используются калорическое уравнение состояния и выражение для определения удельной полной энергии

$$
\begin{gathered}
p=(\gamma-1) \rho U=(\gamma-1) \rho\left[E-0.5\left(u^{2}+v^{2}+w^{2}\right)\right], \\
q=-\lambda \nabla T, \\
U=c_{V} T, \\
E=\frac{p}{(\gamma-1) \rho}+\frac{u^{2}+v^{2}+w^{2}}{2}
\end{gathered}
$$

В уравнениях (6)-(9) $\gamma$ - показатель адиабаты; $U$ - удельная внутренняя энергия; $\lambda$ - коэффициент теплопроводности; $T$ - температура; $c_{V}$ - удельная теплоемкость при постоянном объеме.

Помимо схемы с донорными ячейками в рамках настоящего кода реализован один из вариантов AUSM схем [23]. В соответствии с их общей идеей многомерная задача о распаде произвольного разрыва может быть сведена к аналогичной одномерной задаче. В рамках данного приближения потоки массы импульса и энергии будут зависеть от нормальной к текущей грани компоненты скорости. В ходе вычисления искомых потоковых величин выполняется расщепление числа Маха, давления $p$ и компоненты $p V_{L, K}^{\text {norm }}$, входящей в уравнение энергии. Здесь $V_{L, K}^{\text {norm }}$ - нормальная к грани $K$ элемента $L$ составляющая вектора скорости. В первую очередь определяется изменение потоковых величин за счет давления

$$
\mathbf{F}_{x}=\left[\begin{array}{c}
0 \\
p_{L}^{+}+p_{R}^{-} \\
0 \\
0 \\
0
\end{array}\right], \mathbf{F}_{y}=\left[\begin{array}{c}
0 \\
0 \\
p_{L}^{+}+p_{R}^{-} \\
0 \\
0
\end{array}\right], \mathbf{F}_{z}=\left[\begin{array}{c}
0 \\
0 \\
0 \\
p_{L}^{+}+p_{R}^{-} \\
0
\end{array}\right]
$$

Здесь

$$
p_{L, R}^{ \pm}=\left\{\begin{array}{lll}
\frac{p}{2} \frac{(\mathrm{M} \pm|\mathrm{M}|)}{\mathrm{M}}, & \text { при } & |\mathrm{M}|>1 \\
\frac{p}{4}(\mathrm{M} \pm 1)^{2}(2 \mp \mathrm{M}), & \text { при } & |\mathrm{M}| \leq 1
\end{array}\right.
$$

Значения с индексом $L$ относятся к текущему элементу, а с индексом $R-$ к соседнему через грань $K$ элементу. Компонента $p V_{L, K}^{\text {norm }}$, входящая в уравнение энергии также представляется в виде суммы двух слагаемых $(p V)_{L}^{+}$и $(p V)_{R}^{-}$, которые определяются следующим образом: 


$$
(p V)_{L, R}^{ \pm}= \begin{cases}\frac{p V_{\text {norm }}}{2} \frac{(\mathrm{M} \pm|\mathrm{M}|)}{\mathrm{M}}, & \text { при }|\mathrm{M}|>1 \\ \frac{p}{2}\left(V_{\text {norm }} \pm \mathrm{a}_{1 / 2}\right), & \text { при }|\mathrm{M}| \leq 1\end{cases}
$$

Здесь и далее $\mathrm{a}_{1 / 2}$ - среднее арифметическое значение скорости звука между соседствующими через определенную грань тетраэдральными элементами. Затем определяется поток массы. При этом по аналогии с давлением выполняется расщепление числа Маха

$$
\begin{gathered}
\mathrm{M}_{1 / 2}=\mathrm{M}_{L}^{+}+\mathrm{M}_{R}^{-} \\
\mathrm{M}_{L, R}^{ \pm}=\left\{\begin{array}{l}
\frac{1}{2}(\mathrm{M} \pm|\mathrm{M}|), \text { при }|\mathrm{M}|>1 \\
\pm \frac{1}{4}(\mathrm{M} \pm 1)^{2}, \text { при }|\mathrm{M}| \leq 1
\end{array}\right.
\end{gathered}
$$

Выражение для потока массы через грань $K$ элемента $L$ имеет вид

$$
\begin{gathered}
\Delta \mathrm{m}_{L, K}=\mathrm{a}_{1 / 2} \cdot\left(\rho_{L} \mathrm{M}_{\mathrm{PLUS}}+\rho_{R} \mathrm{M}_{\mathrm{MINUS}}\right) \\
\mathrm{M}_{\mathrm{PLUS}}=\frac{1}{2}\left(\mathrm{M}_{1 / 2}+\left|\mathrm{M}_{1 / 2}\right|\right) \\
\mathrm{M}_{\mathrm{MINUS}}=\frac{1}{2}\left(\mathrm{M}_{1 / 2}-\left|\mathrm{M}_{1 / 2}\right|\right)
\end{gathered}
$$

Через известный поток массы определяются также потоки импульса и энергии. В конечном итоге значения искомых консервативных переменных определяются из интегральных законов сохранения с учетом определенных ранее потоковых величин.

\section{3. Результаты расчетов}

Рассмотренные конфигурации волнолетов отличаются от других подобных ВЛА несколько большим полезным объемом. В зависимости от формы скачка уплотнения, определяющего вид несущей поверхности, в данной статье следует различать ЭК-волнолет (на основе течения за эллиптическим конусом) и НК-волнолет (на основе течения за наклонным круговым конусом). На рис. 1 и рис. 2изображены виртуальные модели данных ВЛА.

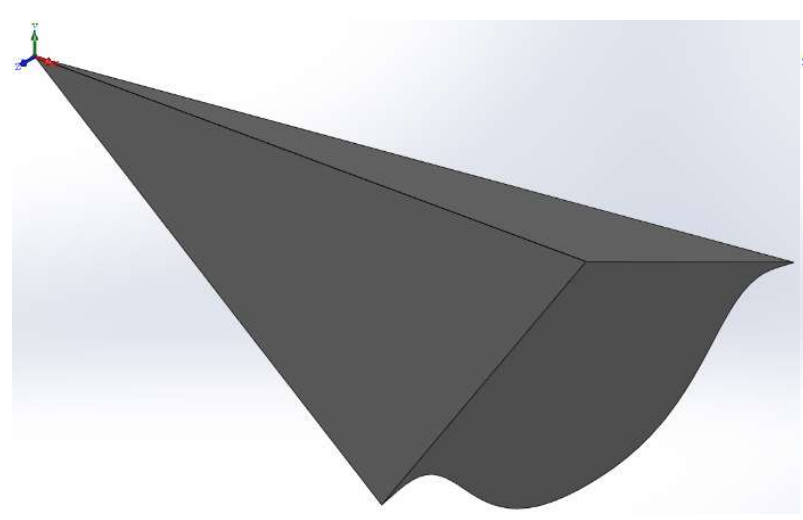

Рис. 1. Виртуальная модель ЭК-Волнолета

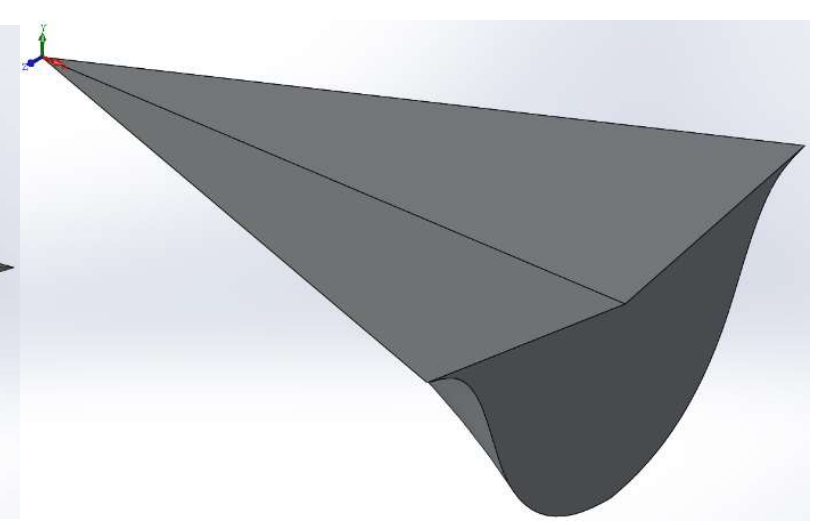

Рис. 2. Виртуальная модель НК-Волнолета

В соответствии с работой [13] несущие поверхности волнолетов могут быть аппроксимированы с помощью следующих соотношений, полученных методами теории малых возмущений 


$$
\begin{gathered}
\frac{\theta}{\delta}=1+0.34 \cdot\left[\tan \left(\frac{\pi}{2}-\frac{\phi}{2}\right)\right]^{12.5} \\
\frac{\theta}{\delta}=1-0.1 \cos (2 \phi)+(0.39+0.1 \cos (2 \phi)) \cdot\left[\tan \left(\frac{\pi-\phi}{2.75}\right)\right]^{7.69}
\end{gathered}
$$

Здесь $\theta$ - полярный угол; $\phi$ - азимутальный угол; $\delta=18.62^{\circ}$ - угол конуса, используемого для генерации невозмущенного поля течения. Выражение (18) описывает нижнюю поверхность НК-волнолета, а выражение (19) соответствует форме поверхности сжатия ЭК-волнолета. В табл. 1 представлены габаритные размеры исследуемых волнолетов, а также значения характерной площади и длины, необходимых для расчета аэродинамических коэффициентов. В табл. 2 содержится информация о параметрах набегающего потока, соответствующих условиям стендового эксперимента [13].

\section{Таблица 1}

Геометрические параметры ЭК- и НК-волнолета

\begin{tabular}{|c|c|c|}
\hline Параметр & ЭК-Волнолет & НК-Волнолет \\
\hline Длина $L$, см & \multicolumn{2}{|c|}{59.995} \\
\hline Ширина $W$, см & 54.610 & 55.829 \\
\hline$S_{r e f}, \mathrm{~cm}^{2}$ & 489.225 & 668.386 \\
\hline$L_{r e f}, \mathrm{~cm}$ & \multicolumn{2}{|c|}{59.995} \\
\hline
\end{tabular}

Таблица 2

Параметры набегающего потока

\begin{tabular}{|c|c|}
\hline Параметр & Значение \\
\hline Давление $p_{\infty}$, эрг $/ \mathrm{cm}^{3}$ & 14530 \\
\hline Плотность $\rho_{\infty}, \Gamma / \mathrm{cm}^{3}$ & $0.546 \times 10^{-4}$ \\
\hline Температура $T_{\infty}, \mathrm{K}$ & 92.8 \\
\hline Число Маха $\mathrm{M}_{\infty}$ & 4 \\
\hline
\end{tabular}

Результаты численного моделирования аэродинамики ЭК- и НК-Волнолета представлены на рис. 3-8. В ходе расчетов использовались неструктурированные тетраэдральные сетки размером до 3 млн. элементов. За исключением лобового сопротивления и аэродинамического качества все графики силовых и моментных характеристик имеют монотонный характер изменения в зависимости от углов атаки или скольжения и вид близкий к линейному распределению. Следует отметить, что для каждой конфигурации может быть определен наиболее оптимальный с точки зрения аэродинамического качества режим полета. В частности для НК-Волнолета такой режим соответствует отрицательному углу атаки $\alpha=-3.72^{\circ}$, что объясняется ориентацией верхней поверхности ВЛА по отношению к набегающему потоку. В целом по характеру изменению аэродинамического качества (рис. 5) может быть сделан вывод о большем потенциале НК-Волнолета именно в отрицательном диапазоне углов атаки. ЭК-Волнолет имеет более резкий характер изменения коэффициента подъемной силы (рис. 3) и в целом большее аэродинамическое сопротивление при всех рассмотренных углах атаки (рис. 4). 


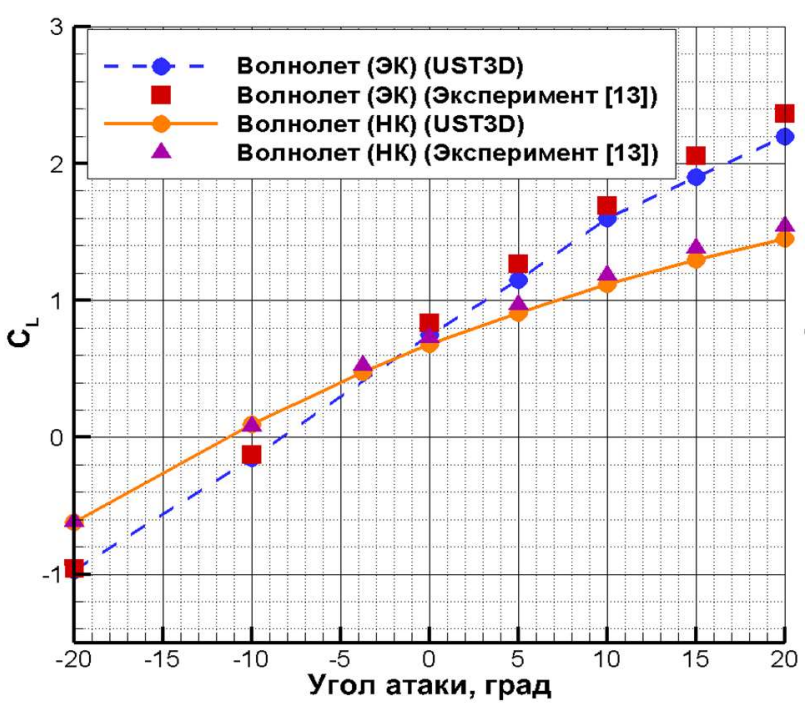

Рис. 3. Коэффициент подъемной силы в зависимости от угла атаки

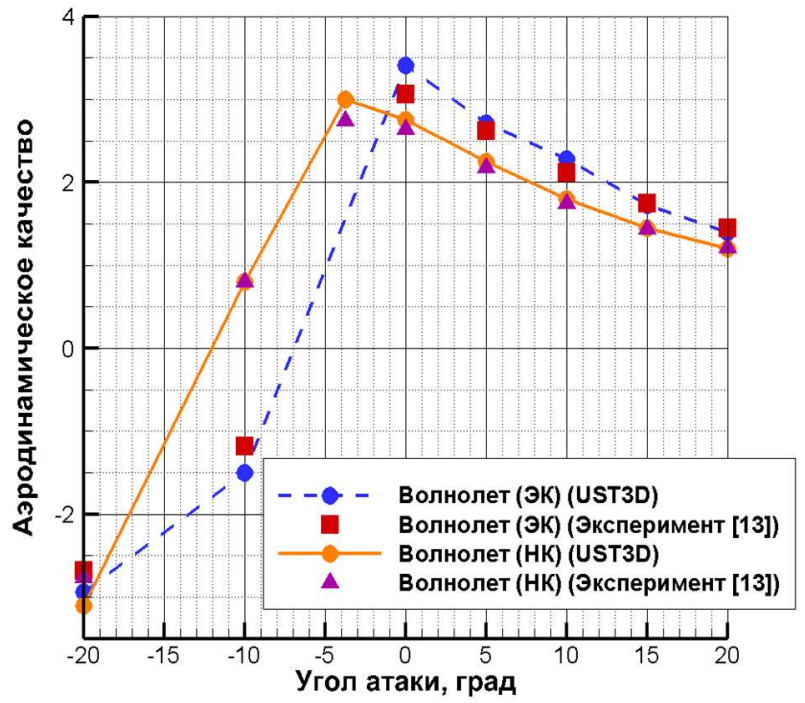

Рис. 5. Аэродинамическое качество в зависимости от угла атаки

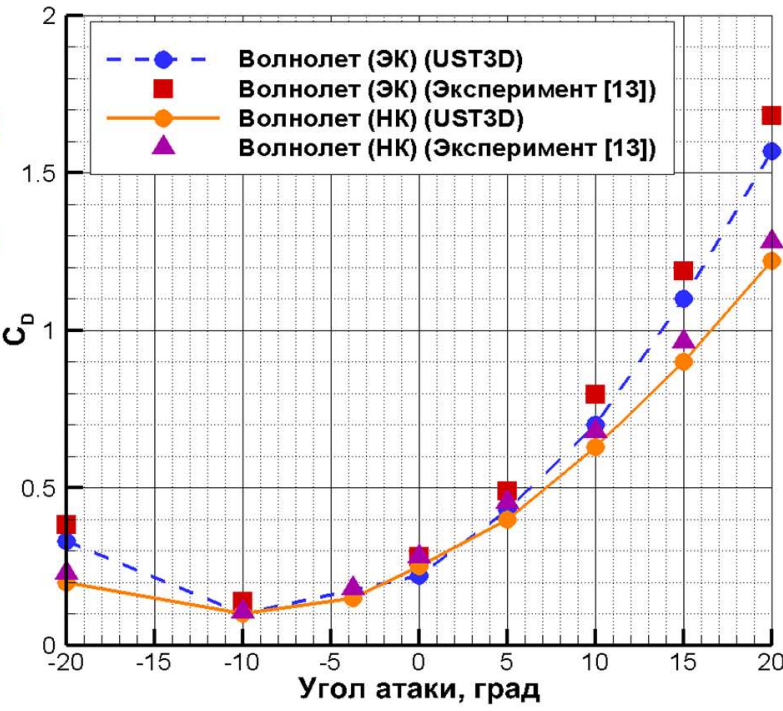

Рис. 4. Коэффициент лобового сопротивления в зависимости от угла атаки

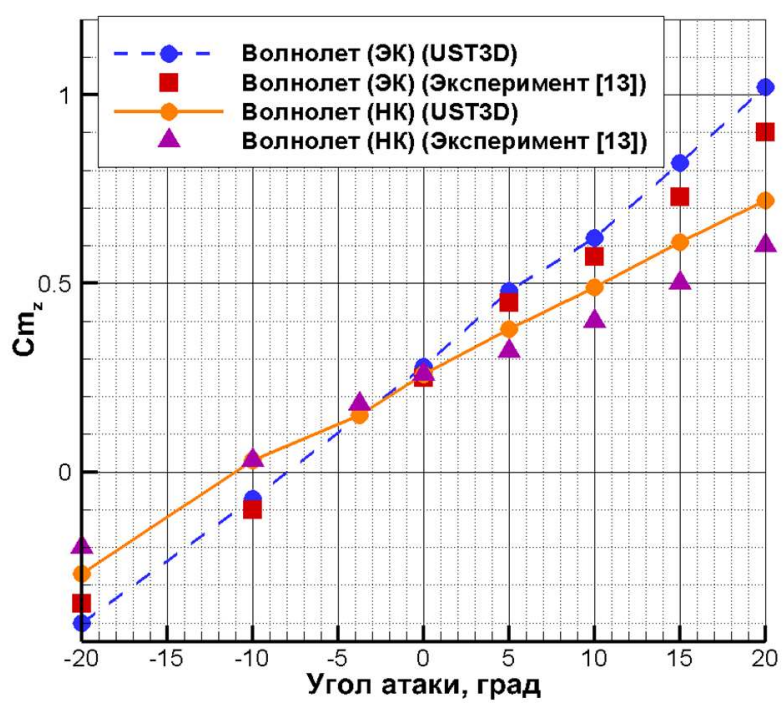

Рис. 6. Коэффициент момента тангажа в зависимости от угла атаки

Определение моментных характеристик позволяет оценить относительное распределение давления по поверхности летательного аппарата и сделать некоторые выводы об его устойчивости в условиях изменения ориентации в полете. Корректная оценка аэродинамических моментов возможна только в условиях определенности положения точки, фиксируемой в эксперименте как центр масс. Положение начала отсчета и ориентация осей координат показаны на рис. 1-2. При расчете аэродинамических моментов принимались следующие координаты характерного центра: для НК-Волнолета $-\mathrm{X}_{Ц . м}=60$ см; $\mathrm{Y}_{\text {ц.м }}=-8.89$ см; для ЭКВолнолета $-\mathrm{X}_{ц . M}=60 \mathrm{~cm} ; \mathrm{Y}_{ц . M}=-6.324 \mathrm{~cm}$.

В целом отмечается удовлетворительное согласие со всеми представленными экспериментальными данными в широком диапазоне углов атаки и скольжения.

Исследование течения в отрывной зоне также имеет существенное фундаментальное значение. При этом процесс моделирования затруднен вихревым характером потока и его нестационарностью. Картина обтекания донного среза существенно зависит от особенностей применяемого численного метода, в частности, от выбора исходных консервативных переменных [17]. 


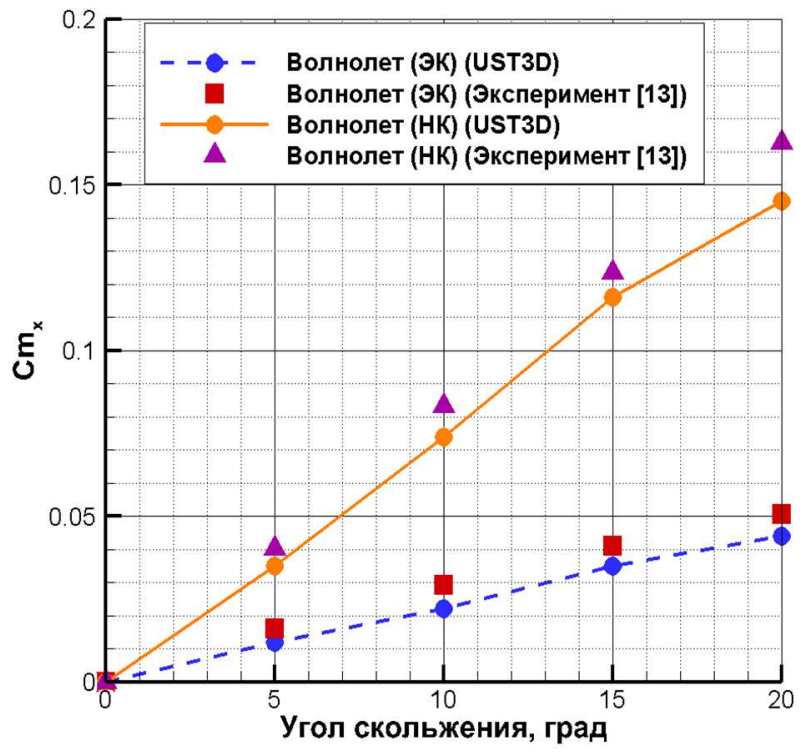

Рис. 7. Коэффициент момента крена в зависимости от угла скольжения

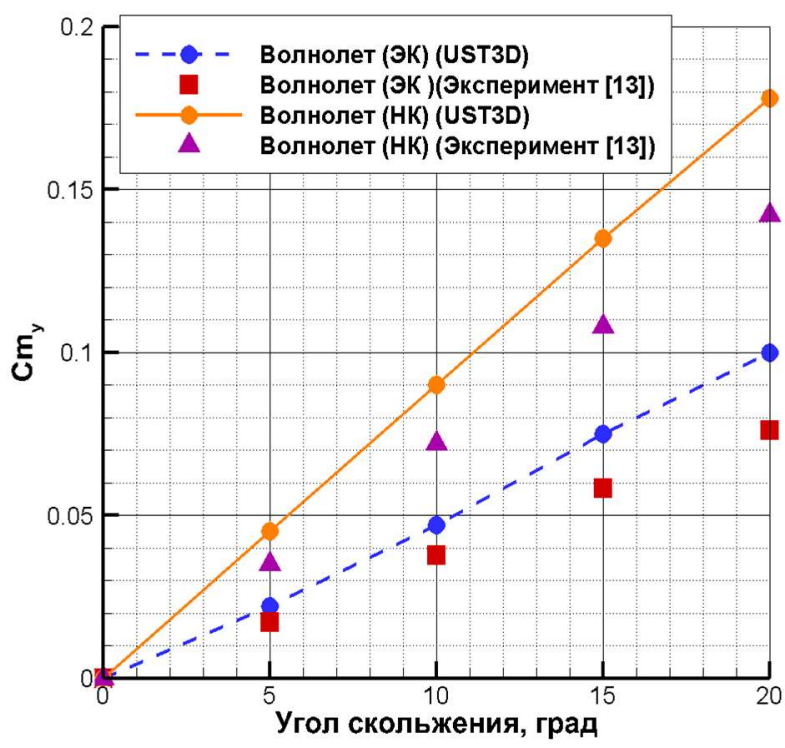

Рис. 8. Коэффициент момента рыскания в зависимости от угла скольжения

В данной статье представлены результаты сравнительных расчетов с использованием разных методов приближенного решения задачи Римана: схемы с донорными ячейками [21] и одного из вариантов AUSM-схемы [23]. Сочетание метода расщепления с различными вариантами решения задачи о распаде произвольного разрыва исследовалось также в работах [24-25]. На рис. 9-10 показаны картины течения в области отрывной зоны ЭК-Волнолета. Характерным эффектом является существенное изменение скоростного режима течения. Отличительной особенностью используемой AUSM-ECUSP схемы является формирование области дозвукового точения в зоне донного среза.

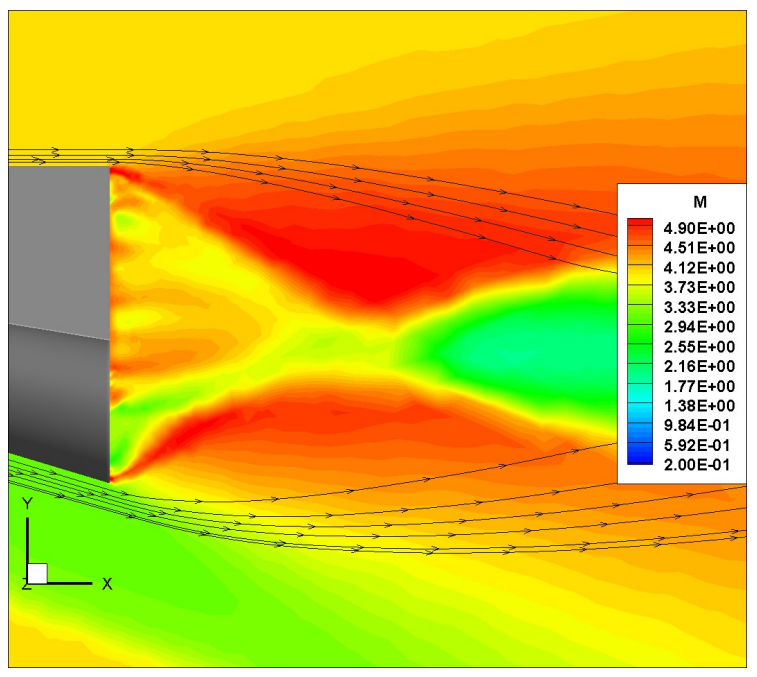

$a$

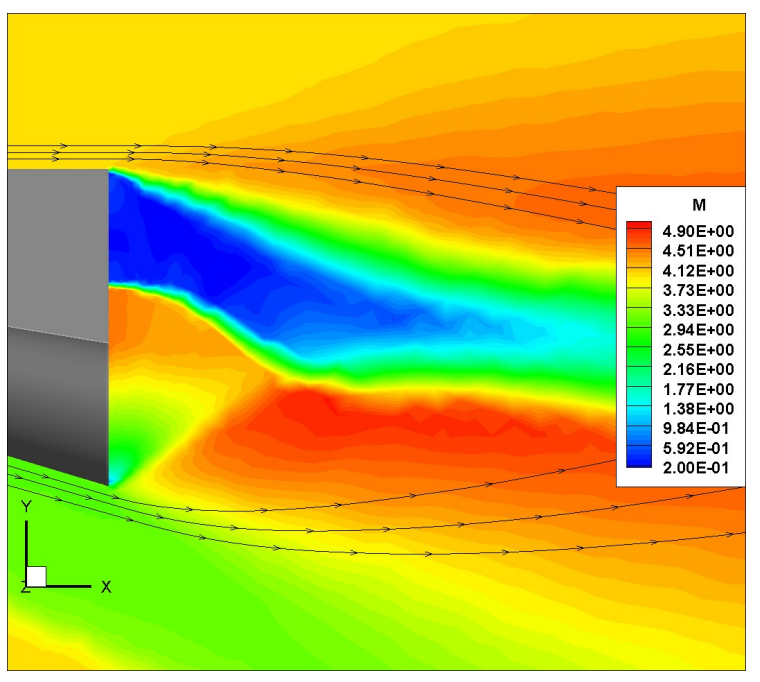

$\sigma$

Рис. 9. Распределение числа Маха в области донного среза ЭК-Волнолета при нулевом угле атаки: a) расчет с применением схемы с донорными ячейками; б) расчет с применением AUSM-ECUSP схемы

При вариации угла атаки, в частности при его увеличении, отмечается сочетание до- и сверхзвуковых зон, что характерно для обеих моделей волнолета (рис. 10). 


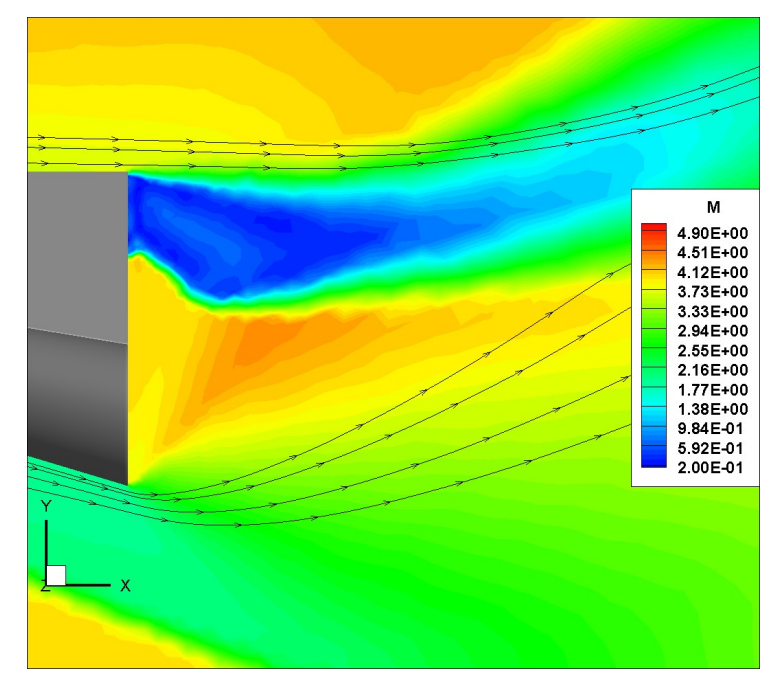

$a$

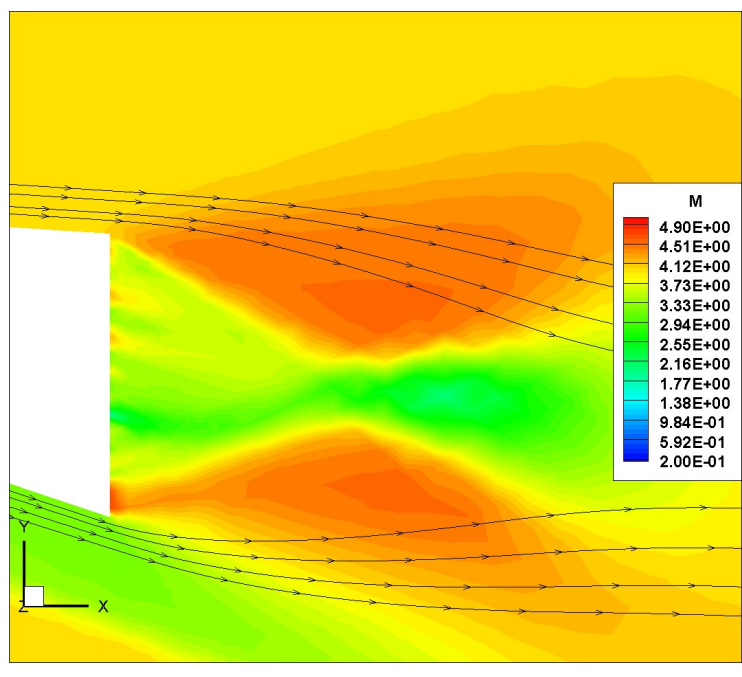

B

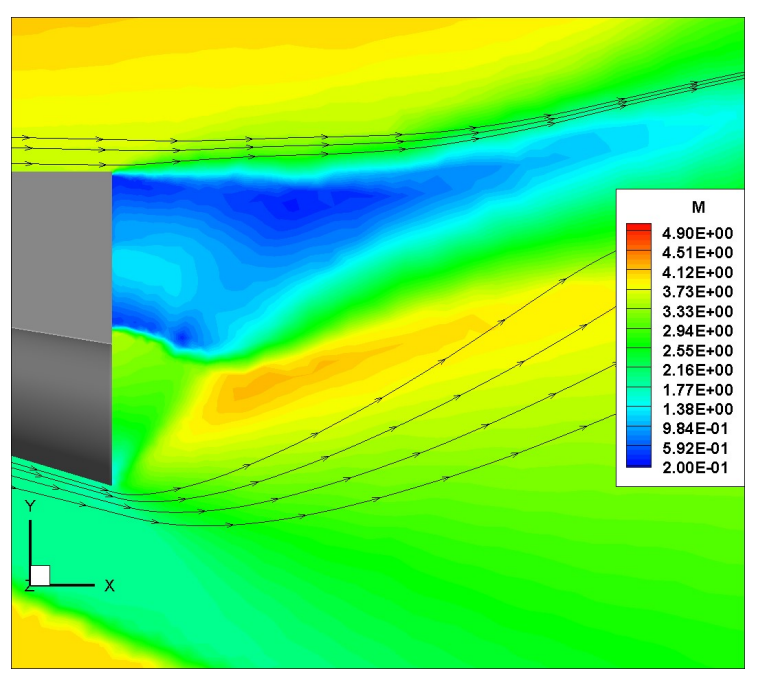

6

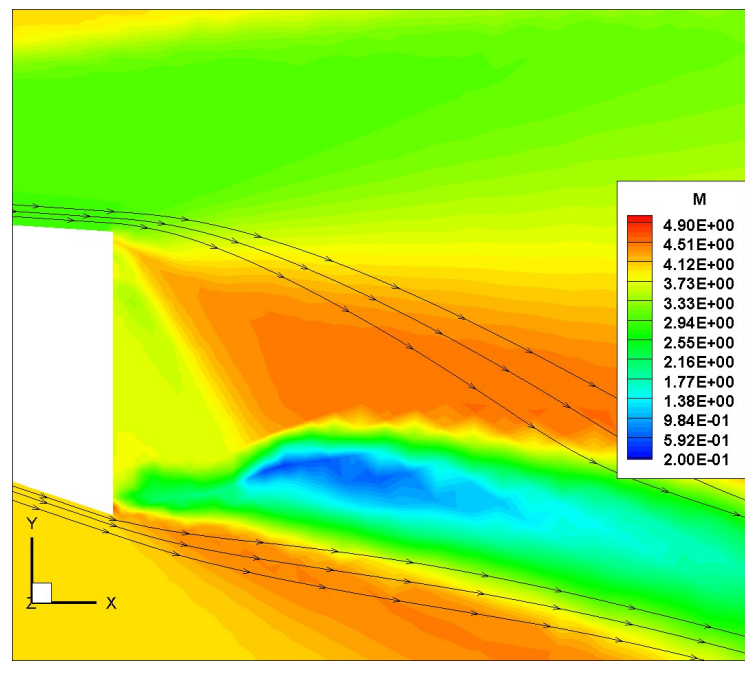

Рис. 10. Распределение числа Маха в области донного среза для: a) ЭК-волнолета - расчет с применением схемы с донорными ячейками при $\alpha=20^{\circ}$; б) ЭК-волнолета - расчет с применением AUSM-ECUSP схемы при $\alpha=20^{\circ} ;$ в) НК-волнолета - расчет с применением схемы с донорными ячейками при $\alpha=-3.72^{\circ}$; г) НК-волнолета - расчет с применением схемы с донорными ячейками при $\alpha=-20^{\circ}$

Заключительным результатом данной работы, отраженным на рис. 11, является распределение коэффициента давления $C_{P}$ в поперечном сечении ЭК-Волнолета, отстоящим от носовой точки ВЛА на расстоянии 39.88 см. При этом начальное значение азимутального угла $\varphi=110^{\circ}$ соответствует острой кромке ЭК-Волнолета, а финальное значение $\varphi=180^{\circ}$ - нижней точке несущей поверхности. Для расчетных случаев, соответствующих углам атаки $\alpha=0^{\circ}$ и $\alpha=5^{\circ}$, было выполнено сравнение расчетных данных с результатами работ [14] и [15], в рамках которых проводилось численное моделирование вязкого обтекания аналогичной модели с использованием структурированных сеток на основе параболизованных уравнений Навье - Стокса, а также в приближении тонкого слоя. Результаты сравнительного анализа говорят о достаточно точном определении распределения коэффициента давления с помощью различных модификаций кода UST3D как по отношению к сторонним расчетным данным, так и к результатам эксперимента [13], несмотря на отсутствие возможности корректного разрешения зоны пограничного слоя. 

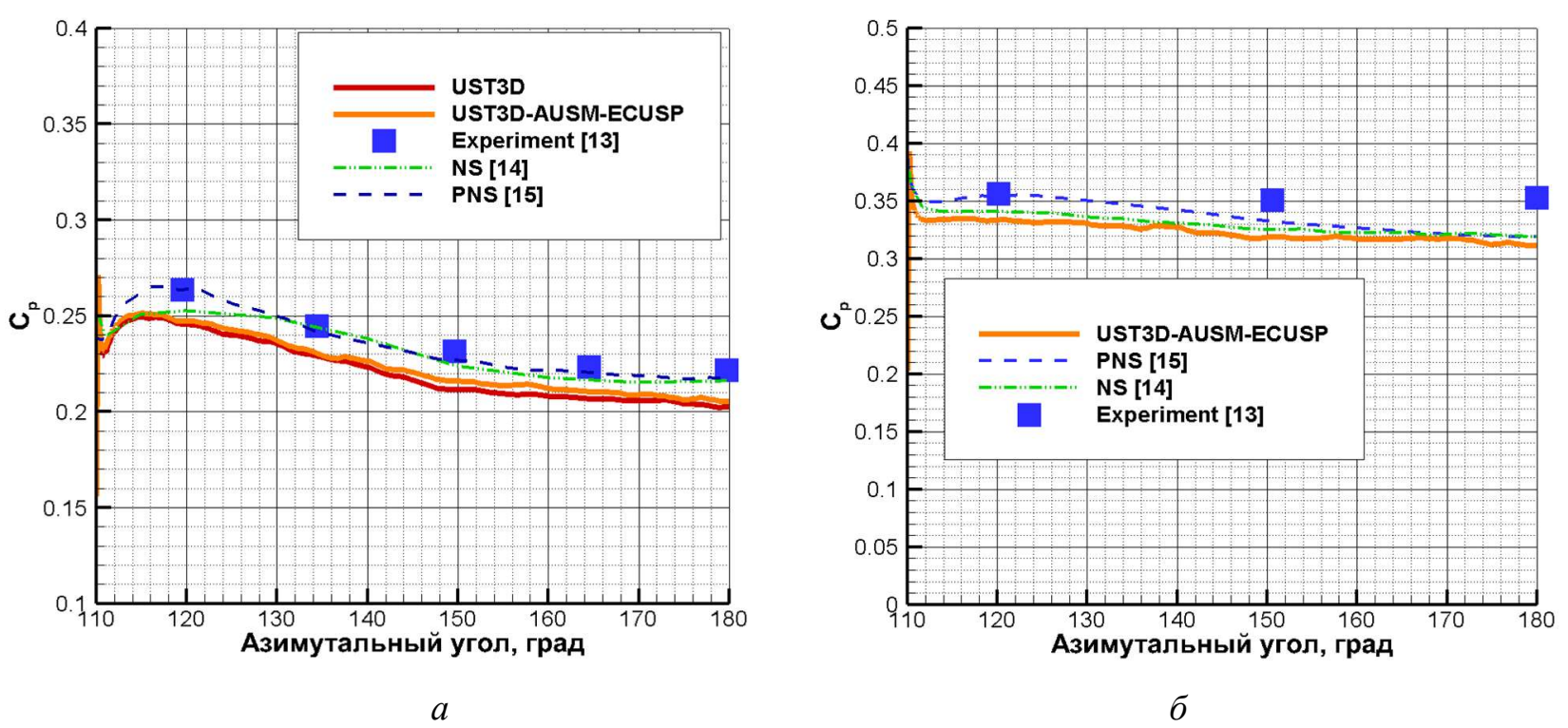

Рис. 11. Распределение коэффициента давления в поперечном сечении ЭК-Волнолета при $\mathrm{M}_{\infty}=4$ : a) сравнение данных для угла атаки $\alpha=0^{\circ}$; б) сравнение данных для угла атаки $\alpha=5^{\circ}$

\section{4. Заключение}

В настоящей работе были представлены результаты расчетов двух конфигураций волнолетов с использованием неструктурированных сеток. На примере сторонних расчетных данных, полученных с применением структурированных сеток, была выполнена верификация компьютерного кода UST3D и его модификации. Важным результатом является также валидация данного компьютерного кода на примере расчета силовых и моментных характеристик ЭК- и НК-Волнолета. Погрешность расчета моментных характеристик может быть обусловлена некоторым несовпадением площади смачиваемой поверхности используемой компьютерной модели волнолета и экспериментального прототипа. В процессе дальнейшего развития компьютерных кодов, использующих неструктурированные сетки, может также представлять интерес применение приближенных методов для оценки поверхностного трения, что позволит достигнуть лучшего понимания структуры общего аэродинамического сопротивления различных летательных аппаратов.

\section{Благодарности}

Работа выполнена в рамках государственного задания № АААА-А20-120011690135-5, а также частично поддержана грантом РФФИ 19-01-00515.

\section{Литература}

1. Bowcutt K.G., Anderson J.D., Capriotti D. Viscous Optimized Hypersonic Waveriders // AIAA Paper 87-0272. 1987. Pp. 1-19. https://doi.org/10.2514/6.1987-272

2. Мажуль И.И. Сравнительный анализ аэродинамики волнолетов, построенных на основе конических и плоских течений // Теплофизика и аэромеханика. 2007. Т. 14. № 1. С. 99-112. http://sibran.ru/journals/issue.php?ID=120101\&ARTICLE ID=126719

3. Воронин В.И., Захарченко В.Ф., Швец А.И. Волнолеты, построенные на течениях за скачками уплотнения в виде эллиптических конусов // ПМТФ. 1994. Т. 4 . № 81. http://sibran.ru/journals/issue.php?ID=119954\&ARTICLE_ID=133534

4. Vanmol D.O., Anderson J.D. Heat Transfer Characteristics of Hypersonic Waveriders with an Emphasis on the Leading Edge Effects//NASA Contractor Report 189586. 1992. $128 \mathrm{p}$. https://ntrs.nasa.gov/archive/nasa/casi.ntrs.nasa.gov/19920012972.pdf 
5. Cockrell Ch.E. Vehicle Integration Effects on Hypersonic Waveriders // NASA-TM-109739. 1994. 132 p. https://ntrs.nasa.gov/archive/nasa/casi.ntrs.nasa.gov/19940029612.pdf

6. Kammeyer M. E., Gillum M. J. Design Validation Tests on a Realistic Hypersonic Waverider at Mach 10, 14 and 16.5 in the Naval Surface Warfare Center Hypervelocity Wind Tunnel No. 9 // NSWCDD/TR-93/198. 1994. 93 p.

7. Lewis M.J., Gillum M.J. Analysis of experimental results on a Mach 14 waverider with blunt leading edges // AIAA Paper 96-0812. 1996. Pp. 1-23. https://doi.org/10.2514/6.1996-812

8. Maikapar G. I. Bodies Formed by the Stream Surfaces of Conical Flows // Fluid Dynamics. Vol. 1. No. 1. 1966. Pp. 126-127. https://doi.org/10.1007/BF01016277

9. Rasmussen M.L. On Hypersonic Flow Past an Unyawed Cone // AIAA Journal. 1967. Vol. 5. No. 8. Pp. 1495-1497. https://doi.org/10.2514/3.4228

10. Rasmussen M. L. Approximation for Hypersonic Flow Past a Slender Elliptic Cone // AIAA Paper 790364. 1979. Pp. 1-11. https://doi.org/10.2514/6.1979-364

11. Rasmussen M. L. Waverider Configurations Derived from Inclined Circular and Elliptic Cones // Journal of Spacecraft and Rockets. 1980. Vol. 17. No. 6. Pp. 537-545. https://doi.org/10.2514/3.57771

12. Yoon B.-h. On-Design Solutions of Hypersonic Flows past Elliptic-Cone Derived Waveriders // KSME Journal Vol. 6. No. 1. 1992. Pp. 24-30. https://doi.org/10.1007/BF02954460

13. Rasmussen M. L. Experimental Forces and Moments on Cone-Derived Waveriders for $\mathrm{M}=3$ to 5 // Journal of Spacecraft and Rockets. 1982. Vol. 19. No. 6. Pp. 592-598. https://doi.org/10.2514/3.62306

14. Liao J.-R., Isaac K.M., Miles J.B., Tsai B.-J. Navier-Stokes Simulation for Cone-Derived Waverider // AIAA Journal. 1992. Vol. 30. No. 6. Pp. 1521-1528. https://doi.org/10.2514/3.11096

15. Kato H., Tannehill J.C. Numerical Calculation of Viscous Flow Over Hypersonic Waveriders // AIAA Paper 97-2292. 1997. Pp. 1-11. https://doi.org/10.2514/6.1997-2292

16. Surzhikov S.T. Validation of computational code UST3D by the example of experimental aerodynamic data // Journal of Physics: Conference Series. 2017. Vol. 815. No 12023. https://doi.org/10.1088/1742$\underline{6596 / 815 / 1 / 012023}$

17. Surzhikov S.T. Comparative Analysis of the Results of Aerodynamic Calculation of a Spherical Blunted Cone on a Structured and Unstructured Grid // Journal of Physics: Conference Series. 2019. Vol. 1250. No 012007 https://doi.org/10.1088/1742-6596/1250/1/012007

18. Yatsukhno D.S. Computational study of the waverider aerothermodynamics by the UST3D computer code// Journal of Physics: Conference Series. 2018. Vol. 1009. No. 012002. https://doi.org/10.1088/1742-6596/1009/1/012002

19. Яцухно Д. С., Суржиков С. Т. Метод расщепления по физическим процессам в задаче моделирования обтекания перспективного высокоскоростного летательного аппарата//Вестник МГТУ им. Н.Э. Баумана. Сер. «Машиностроение». 2018. № 1. С. 20-33.

20. Kryuchkova A.S. Development and testing of non-viscid solver based on UST3D programming code // Journal of Physics: Conference Series. 2019. Vol. 1250. No. 012009. https://doi.org/10.1088/1742$\underline{6596 / 1250 / 1 / 012009}$

21. Jentry R. A., Martin R. E., Daly B. J. An Eulerian Differencing Method for Unsteady Compressible Flow Problems//J. Comput. Phys. 1966. Vol. 1. No. 1. pp. 87-118. https://doi.org/10.1016/00219991(66)90014-3

22. Shyy, W., Thakur, S.S., Ouyang, H., Liu, J., and Blosch, E. Computational Techniques for Complex Transport Phenomena. 1997. Cambridge University Press. 321 p.

23. Hu Z., Zha G. Simulation of 3D Flows of Propulsion Systems Using an Efficient Low Diffusion E-CUSP Upwind Scheme // AIAA Paper 2004-4082. 2004. Pp. 1-12. https://doi.org/10.2514/6.2004$\underline{4082}$

24. Железнякова А. Л., Суржиков С. Т. Расчет гиперзвукового обтекания тел сложной формы на неструктурированных тетраэдральных сетках с использованием схемы AUSM // TBT. 2014. Том 52. выпуск 2. С. 283-293. https://doi.org/10.7868/S0040364414020215 
25. Сильвестров П.В., Суржиков С.Т. Расчет аэротермодинамики высокоскоростного летательного аппарата X-43 с использованием компьютерных кодов UST3D и UST3D-AUSMPW//Физикохимическая кинетика в газовой динамике. 2019. Том 20, выпуск 4. http://chemphys.edu.ru/is$\underline{\text { sues/2019-20-4/articles/865 }}$

\section{References}

1. Bowcutt, K. G., Anderson, J. D., Capriotti, D., "Viscous Optimized Hypersonic Waveriders," 25th AIAA Aerospace Sciences Meeting, AIAA Paper 87-0272, 1987. https://doi.org/10.2514/6.1987-272

2. Mazhul, I. I., "Sravnitel'nyj analiz ajerodinamiki volnoletov, postroennyh na osnove konicheskih i ploskih techenij," Teplofizika i ajeromehanika, Vol. 14, No. 1, 2007, pp. 99-112.

http://sibran.ru/journals/issue.php?ID=120101\&ARTICLE_ID=126719

3. Voronin, V. I., Zakharchenko, V. F., Shvets, A. I., "Volnolety, postroennye na techenijah za skachkami uplotnenija v vide jellipticheskih konusov," PMTF, No.4, 1994, pp. 81-87. http://sibran.ru/journals/issue.php?ID=119954\&ARTICLE_ID=133534

4. Vanmol, D. O., Anderson, J. D., "Heat Transfer Characteristics of Hypersonic Waveriders with an Emphasis on the Leading Edge Effects," NASA Contractor Report 189586, 1992.

https://ntrs.nasa.gov/archive/nasa/casi.ntrs.nasa.gov/19920012972.pdf

5. Cockrell, Ch. E., "Vehicle Integration Effects on Hypersonic Waveriders," NASA-TM-109739, 1994. https://ntrs.nasa.gov/archive/nasa/casi.ntrs.nasa.gov/19940029612.pdf

6. Kammeyer, M. E., Gillum, M. J., "Design Validation Tests on a Realistic Hypersonic Waverider at Mach 10, 14 and 16.5 in the Naval Surface Warfare Center Hypervelocity Wind Tunnel No. 9," NSWCDD/TR-93/198, 1994.

7. Lewis, M. J., Gillum, M. J., "Analysis of Experimental Results on a Mach 14 Waverider with Blunt Leading Edges," 34th Aerospace Sciences Meeting and Exhibit, AIAA Paper 96-0812, Jan. 1996. https://doi.org/10.2514/6.1996-812

8. Maikapar, G. I., "Bodies Formed by the Stream Surfaces of Conical Flows," Fluid Dynamics, Vol. 1, No. 1, 1966, pp. 126-127. https://doi.org/10.1007/BF01016277

9. Rasmussen, M. L., "On Hypersonic Flow Past an Unyawed Cone,” AIAA Journal, Vol. 5, No. 8, 1967, pp. 1495-1497. https://doi.org/10.2514/3.4228

10. Rasmussen, M. L., “Approximation for Hypersonic Flow Past a Slender Elliptic Cone," 17th Aerospace Sciences Meeting, AIAA Paper 79-0364, Jan. 1979. https://doi.org/10.2514/6.1979-364

11. Rasmussen, M. L., "Waverider Configurations Derived from Inclined Circular and Elliptic Cones," Journal of Spacecraft and Rockets, Vol. 17, No. 6, 1980, pp. 537-545. https://doi.org/10.2514/3.57771

12. Yoon, B.-h., "On-Design Solutions of Hypersonic Flows past Elliptic-Cone Derived Waveriders," KSME Journal, Vol. 6, No. 1, 1992, pp. 24-30. https://doi.org/10.1007/BF02954460

13. Rasmussen, M. L., "Experimental Forces and Moments on Cone-Derived Waveriders for $\mathrm{M}=3$ to 5," Journal of Spacecraft and Rockets, Vol. 19, No. 6, 1982, pp. 592-598. https://doi.org/10.2514/3.62306

14. Liao, J.-R., Isaac, K. M., Miles, J. B., Tsai, B.-J., "Navier-Stokes Simulation for Cone-Derived Waverider," AIAA Journal, Vol. 30, No. 6, 1992, pp. 1521-1528. https://doi.org/10.2514/3.11096

15. Kato, H., Tannehill, J.C., "Numerical Calculation of Viscous Flow Over Hypersonic Waveriders," AIAA Paper 97-2292, 1997, pp. 1-11. https://doi.org/10.2514/6.1997-2292

16. Surzhikov, S. T., "Validation of computational code UST3D by the example of experimental aerodynamic data," Journal of Physics: Conference Series, Vol. 815, No. 12023, 2017. https://doi.org/10.1088/1742-6596/815/1/012023

17. Surzhikov, S. T., "Comparative Analysis of the Results of Aerodynamic Calculation of a Spherical Blunted Cone on a Structured and Unstructured Grid," Journal of Physics: Conference Series, Vol. 1250, No. 012007, 2019. https://doi.org/10.1088/1742-6596/1250/1/012007 
18. Yatsukhno, D. S., "Computational study of the waverider aerothermodynamics by the UST3D computer code," Journal of Physics: Conference Series, Vol. 1009, No. 012002, 2018.

https://doi.org/10.1088/1742-6596/1009/1/012002

19. Yatsukhno, D. S, Surzhikov, S. T., "Metod rasshheplenija po fizicheskim processam v zadache modelirovanija obtekanija perspektivnogo vysokoskorostnogo letatel'nogo apparata," Herald of the Bauman Moscow State Technical University: Mechanical Engineering, No. 1, 2018, pp. 20-33.

20. Kryuchkova, A.S., Development and testing of non-viscid solver based on UST3D programming code," Journal of Physics: Conference Series, Vol. 1250, No. 012009, 2019.

https://doi.org/10.1088/1742-6596/1250/1/012009

21. Jentry, R. A., Martin, R. E., Daly, B. J., "An Eulerian Differencing Method for Unsteady Compressible Flow Problems," J. Comput. Phys, Vol. 1, No. 1, 1966, pp. 87-118.

https://doi.org/10.1016/0021-9991(66)90014-3

22. Shyy, W., Thakur, S. S., Ouyang, H., Liu, J., and Blosch, E., Computational Techniques for Complex Transport Phenomena, Cambridge University Press, 1997, 321 p.

23. $\mathrm{Hu}, \mathrm{Z}$., Zha, G., "Simulation of 3D Flows of Propulsion Systems Using an Efficient Low Diffusion ECUSP Upwind Scheme," 40th AIAA/ASME/SAE/ASEE Joint Propulsion Conference and Exhibit, AIAA Paper 2004-4082, July 2004. https://doi.org/10.2514/6.2004-4082

24. Zheleznyakova, A. L., Surzhikov, S. T., "Calculation of a hypersonic flow over bodies of complex configuration on unstructured tetrahedral meshes using the AUSM scheme," High Temperature, , Vol. 52, No. 2, 2014, pp. 271-281. https://doi.org/10.1134\%2FS0018151X14020217

25. Silvestrov, P. V., Surzhikov, S. T., "Calculation of aerothermodynamics for high-speed aircraft X-43 using computer code UST3D and UST3D-AUSMPW," Physical-Chemical Kinetics in Gas Dynamics, Vol. 20, No. 4, 2019. http://chemphys.edu.ru/issues/2019-20-4/articles/865

Статья поступила в редакцию 15 мая 2020 г. 